\title{
Applying the Shadow Photography and Interferometric Particle Imaging Methods for Determining the Integral Characteristics of Water Droplet Evaporation in Hot Combustion Products of Typical Liquid Flammable Substances
}

\author{
Alena O. Zhdanova, Pavel A. Strizhak, Maxim V. Piskunov \\ Institute of Power Engineering, National Research Tomsk Polytechnic University \\ 30, Lenin Avenue, Tomsk, Russia \\ zhdanovaao@tpu.ru; pavelspa@tpu.ru; piskunovmv@tpu.ru
}

\begin{abstract}
The role of hot gas-vapor-droplet flows in promising technologies is enhanced. They are such technologies as constructing technologies of heat carriers based on the stack effluents, droplets and vapors of water; thermal or flame purification of liquids, emulsions and suspensions; processing the sluggy thermally loaded surfaces of power equipment; defrosting the granular medium; polydisperse extinguishing. The periods of heating or cooling various surfaces can be significantly minimized by hot gas-vapor-droplet flows. The work presents the investigation results of the evaporation features of water droplets while they're moving through hot combustion products of typical liquid flammable substances by the Shadow Photography and Interferometric Particle Imaging methods. The integral characteristics of motion and evaporation are defined. Initial sizes of droplets and temperatures of combustion products are varied within wide ranges: $0.05-4 \mathrm{~mm}$ and $400-1900 \mathrm{~K}$. The conditions, under which it is possible almost completely or fairly moderate (with minimal change in mass of the liquid) evaporation of droplets under intense heat, are determined.
\end{abstract}

Keywords: water droplet, aerosol, evaporation, hot combustion products, flammable liquids

\section{Introduction}

In the last decade, there is a tendency to strengthen the role of hot $(1000 \mathrm{~K})$ gas-vapor-droplet flows for modern heat technologies [1-3]. It is possible to highlight the promising hot gas-vapor-droplet techniques (thermal or flame purification of liquid, heat carriers based on the stack effluents, droplets and vapors of water, polydisperse extinguishing). In recent years, non-contact diagnostic methods of two-phase and heterogeneous gas-vapor-droplet flows are considered to the most efficient ones [4-8]. It is interesting to analyze the possibility of using methods of Shadow Photography (SP) and Interferometric Particle Imaging (IPI) for conditions of high (more than $1000 \mathrm{~K}$ ) temperatures of gases.

\section{Experimental setup and procedure}

For experimentations we used the setup with a high-speed system of video recording and cross-correlation software (fig. 1).

In the main components, the setup is similar to that used in the experiments $[9,10]$. We installed the following devices as registration equipment: synchronizing processor 17 (maximum sampling signals - $10 \mathrm{~ns}$ ), double pulse Nd:YAG laser 18 (wavelength $-532 \mathrm{~nm}$, minimum energy per pulse $-70 \mathrm{~mJ}$, maximum pulse duration $-12 \mathrm{~ns}$, repetition rate $-15 \mathrm{~Hz}$ ), CCD video cameras "IMPERX IGV B2020M" 3, 4 (image format - 2048×2048 pixels, frame rate - not less than $1.5 \mathrm{~Hz}$, minimum delay between two consecutive frames $-5 \mu \mathrm{s}$ ), and high-speed CMOS cameras "Phantom V411" 1 and "Phantom MIRO M310" 2 (image format $-1280 \times 1280$ pixels, maximum frame rate $-6 \cdot 10^{5} \mathrm{fps}$ ). 


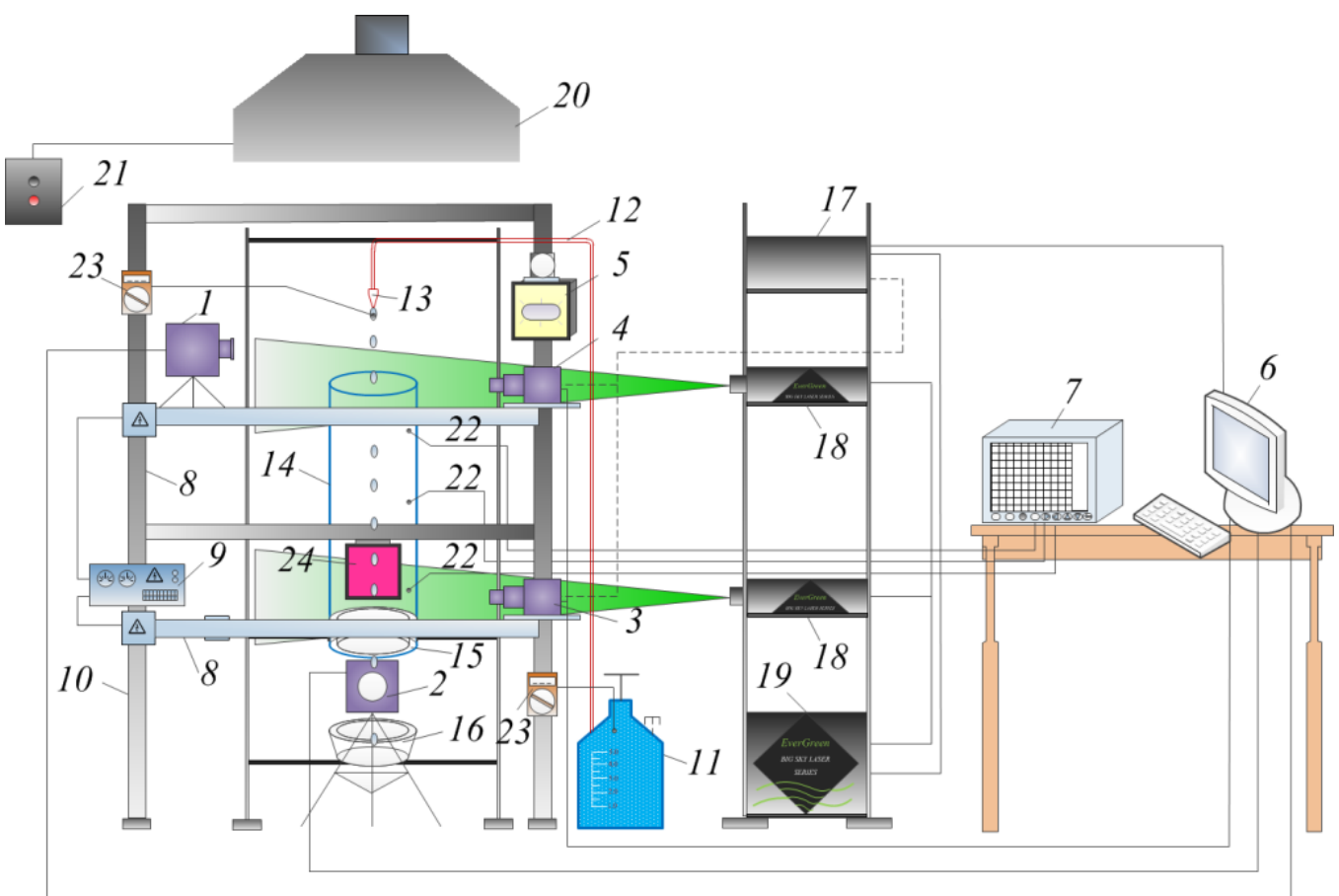

Fig. 1: Experimental setup: 1,2 - high-speed video cameras; 3, 4- cross-correlation video cameras; 5 - spotlight; 6 - personal computer; 7 - technological multichannel registrar (RMT); 8 - motorized positioning device; 9 - power supply for motorized positioning device; 10 - aluminum rack; 11 - container with water; 12 - water supply channel; 13 - spray/dosing device; 14 - quartz

cylinder; 15 - hollow cylinder with a flammable liquid; 16 - water catcher; 17 - synchronizer for a personal computer, crosscorrelation cameras, and laser; 18 - double pulse Nd:YAG laser; 19 - laser line generator; 20 - air flow system; 21 - remote on/off of air flow system; 22 - type A-1 thermocouple; 23 - digital temperature meter with type L thermocouples; 24 - diffusion screen with a light guide.

The setup used in the present work differs from experimental equipment with additional sets of devices for the implementation of panoramic diagnostic methods of flows as Stereoscopic Particle Image Velocimetry (Stereo PIV), Particle Tracking Velocimetry (PTV), Shadow Photography (SP). Their application allows us to register simultaneously such parameters of the investigated processes as the temperature of water $T_{\mathrm{w}}$ and the gaseous medium $T_{\mathrm{g}}$, the velocity $U_{\mathrm{d}}$ and sizes $R_{\mathrm{d}}$ of water droplets, and the velocity $U_{\mathrm{g}}$ of counter hot gas flow. Conversely, this accelerated significantly processing the results of experiments and minimized the discrepancy between the values of the recorded parameters (it is inevitable during duplication series of experiments).

We carried out the experimental investigations in two steps. In the first stage, the experiments were conducted with single large $\left(R_{\mathrm{d}}=1-3 \mathrm{~mm}\right)$ water droplets. The second step included the experiments with atomized water flows (size of droplets was $R_{\mathrm{d}}=0.05-0.35 \mathrm{~mm}$ ). Each step involved four series of experiments. We passed water droplets through the hot gas medium formed by combustion products of typical liquid flammable substance (gasoline AI-92, kerosene TS-1, acetone - dimethyl ketone, National State Standard 2768-84, technical alcohol - ethyl synthetic, National State Standard R 525742006). Temperature of combustion products $T_{\mathrm{g}}$ in a cylinder 14 was monitored at different points in height and its axis of symmetry similar to the experiments $[9,10]$. Water droplets were supplied from the dosing device (atomizer) 13 into internal area of cylinder 14. Concurrently, we started the procedure of photo and video recording of the process of water droplet motion. Shooting was carried out simultaneously in two registration areas: at the inlet and outlet from the cylinder 14.

\section{Results and discussion}

Resulting the experimental investigation results processing and subsequent analysis, we obtained the dependences of parameter $\Delta R$ on initial sizes of $R_{\mathrm{d}}$ (table 1), speed $U_{\mathrm{d}}$ (table 2) and initial temperature $T_{\mathrm{w}}$ (table 3 ) of water droplets. To calculate $\Delta R$, the values of droplet sizes at the inlet into a cylinder with hot gases and at the outlet from it were used. The length of the path of droplet movement in a flow of combustion products was $1 \mathrm{~m}$. 
Table 1: Dependencies of the $\Delta R$ parameter on the initial size $R_{\mathrm{d}}$ of single droplets passing the distance of $1 \mathrm{~m}$ in high-temperature gasoline combustion products.

\begin{tabular}{|c|c|c|c|}
\hline & $U_{\mathrm{d}} \approx 2 \mathrm{~m} / \mathrm{s}$ & $U_{\mathrm{d}} \approx 3 \mathrm{~m} / \mathrm{s}$ & $U_{\mathrm{d}} \approx 3.5 \mathrm{~m} / \mathrm{s}$ \\
\hline$R_{\mathrm{d}} \approx 2 \mathrm{~mm}$ & 0,15 & 0,09 & 0,08 \\
\hline$R_{\mathrm{d}} \approx 2,5 \mathrm{~mm}$ & 0,13 & 0,06 & 0,05 \\
\hline$R_{\mathrm{d}} \approx 3 \mathrm{~mm}$ & 0,11 & 0,04 & 0,04 \\
\hline
\end{tabular}

Varying the initial speed of droplets within the wide range (significantly affects the residence time of droplets in the hot gas medium) allowed also allocating the conditions (table 2) when the evaporation process is characterized by minimal values of $\Delta R$.

Table 2: The dependencies of the $\Delta R$ parameter on the initial velocity $U_{\mathrm{d}}$ of single droplets passing the distance of $1 \mathrm{~m}$ in hightemperature gasoline combustion products.

\begin{tabular}{|c|c|c|c|}
\hline & $R_{\mathrm{d}} \approx 2.3 \mathrm{~mm}$ & $R_{\mathrm{d}} \approx 2.5 \mathrm{~mm}$ & $R_{\mathrm{d}} \approx 2.8 \mathrm{~mm}$ \\
\hline$U_{\mathrm{d}} \approx 2 \mathrm{~m} / \mathrm{s}$ & 0,15 & 0,14 & 0,13 \\
\hline$U_{\mathrm{d}} \approx 2.5 \mathrm{~m} / \mathrm{s}$ & 0,11 & 0,09 & 0,08 \\
\hline$U_{\mathrm{d}} \approx 3 \mathrm{~m} / \mathrm{s}$ & 0,09 & 0,07 & 0,06 \\
\hline$U_{\mathrm{d}} \approx 3.5 \mathrm{~m} / \mathrm{s}$ & 0,07 & 0,05 & 0,04 \\
\hline$U_{\mathrm{d}} \approx 4 \mathrm{~m} / \mathrm{s}$ & 0,06 & 0,04 & 0,03 \\
\hline
\end{tabular}

We established the extent of the influence of the droplet velocity on the integral characteristics of evaporation. This also confirms the decisive influence of the inertia of liquid heating up on the conditions of its evaporation. On the one hand, the growth of droplet velocity should lead to the enhancement of heat transfer on the "liquid - gas" boundary. Estimates show that the increase in droplet velocity from $1 \mathrm{~m} / \mathrm{s}$ to $5 \mathrm{~m} / \mathrm{s}$ can result in an increase of the heat transfer coefficient between the droplet and gas in several times. It should intensify the evaporation of a liquid and lead to the rise of the $\Delta R$ values. However, table 2 , indicates the opposite effect ( $\Delta R$ values decrease with increasing $U_{\mathrm{d}}$ ). This result can be explained by the fact that with the growth of droplet velocity $U_{\mathrm{d}}$, the thickness of its subsurface layer reduces. A droplet heats up during the motion through high-temperature gas. By reducing the initial droplet velocity, there is an increase in the characteristic times of the presence of droplets in the high-temperature area, and of heating up the subsurface layer of a liquid. Accordingly, there is an increase in the mass of a liquid evaporated during the motion in a gas. At relatively small times (up to $1 \mathrm{~s}$ ) of the motion of liquid droplets through high-temperature gases and $U_{\mathrm{d}} \approx U_{\mathrm{g}}$, the influence of the initial droplet velocity is quite moderate. If $U_{\mathrm{d}}>U_{\mathrm{g}}$, this effect intensifies.

The evaporation of water droplets while moving through the hot gases (it is particularly important for a short duration of droplet movement through the combustion products) can be accelerated by several times due to their preheating (table 3 ).

Table 3: The dependencies of the $\Delta R$ parameter on the initial temperature $T_{\mathrm{w}}$ of single water droplets passing the distance of $1 \mathrm{~m}$ in high-temperature gasoline combustion products.

\begin{tabular}{|c|c|c|c|}
\hline & $R_{\mathrm{d}} \approx 2.3 \mathrm{~mm}$ & $R_{\mathrm{d}} \approx 2.5 \mathrm{~mm}$ & $R_{\mathrm{d}} \approx 2.8 \mathrm{~mm}$ \\
\hline$T_{\mathrm{w}} \approx 290 \mathrm{~K}$ & 0,06 & 0,05 & 0,05 \\
\hline$T_{\mathrm{w}} \approx 300 \mathrm{~K}$ & 0,07 & 0,06 & 0,052 \\
\hline$T_{\mathrm{w}} \approx 310 \mathrm{~K}$ & 0,08 & 0,065 & 0,055 \\
\hline$T_{\mathrm{w}} \approx 320 \mathrm{~K}$ & 0,09 & 0,07 & 0,06 \\
\hline$T_{\mathrm{w}} \approx 330 \mathrm{~K}$ & 0,10 & 0,075 & 0,065 \\
\hline$T_{\mathrm{w}} \approx 340 \mathrm{~K}$ & 0,12 & 0,08 & 0,07 \\
\hline$T_{\mathrm{w}} \approx 350 \mathrm{~K}$ & 0,13 & 0,09 & 0,073 \\
\hline
\end{tabular}


The analysis of table 3 shows that preheating the liquid intensifies its evaporation even under relatively high speeds.

The conducted experiments with a flow of droplets at their sizes less than $350 \mu \mathrm{m}$ allowed establishing significantly higher values of the parameter $\Delta R$ (table 4-6) compared with large droplets (0.5-4 mm). As a consequence, it is possible to allocate a significant extent of the impact of the considered factors (the initial speed, the size and the temperature droplets) on the conditions of vaporization.

Table 4: The dependencies of the $\Delta R$ parameter on the initial droplet sizes $R_{\mathrm{m}}$ of the atomized water flow passing the distance of $1 \mathrm{~m}$ in gasoline combustion products.

\begin{tabular}{|c|c|c|c|}
\hline & $U_{\mathrm{m}} \approx 1 \mathrm{~m} / \mathrm{s}$ & $U_{\mathrm{m}} \approx 1.5 \mathrm{~m} / \mathrm{s}$ & $U_{\mathrm{m}} \approx 2 \mathrm{~m} / \mathrm{s}$ \\
\hline$R_{\mathrm{m}} \approx 0.05 \mathrm{~mm}$ & 1 & 1 & 0,92 \\
\hline$R_{\mathrm{m}} \approx 0.1 \mathrm{~mm}$ & 1 & 0,99 & 0,73 \\
\hline$R_{\mathrm{m}} \approx 0.15 \mathrm{~mm}$ & 0,86 & 0,79 & 0,62 \\
\hline$R_{\mathrm{m}} \approx 0.2 \mathrm{~mm}$ & 0,74 & 0,68 & 0,54 \\
\hline$R_{\mathrm{m}} \approx 0.25 \mathrm{~mm}$ & 0,65 & 0,59 & 0,49 \\
\hline$R_{\mathrm{m}} \approx 0.3 \mathrm{~mm}$ & 0,59 & 0,54 & 0,44 \\
\hline$R_{\mathrm{m}} \approx 0.35 \mathrm{~mm}$ & 0,54 & 0,49 & 1 \\
\hline
\end{tabular}

Table 5: The dependencies of the $\Delta R$ parameter on the initial droplet velocity $U_{\mathrm{m}}$ of the atomized water flow passing the distance of 1 $\mathrm{m}$ in gasoline combustion products.

\begin{tabular}{|c|c|c|c|}
\hline & $R_{\mathrm{m}} \approx 0.15 \mathrm{~mm}$ & $R_{\mathrm{m}} \approx 0.25 \mathrm{~mm}$ & $R_{\mathrm{m}} \approx 0.35 \mathrm{~mm}$ \\
\hline$U_{\mathrm{m}} \approx 1 \mathrm{~m} / \mathrm{s}$ & 0,86 & 0,66 & 0,55 \\
\hline$U_{\mathrm{m}} \approx 1.2 \mathrm{~m} / \mathrm{s}$ & 0,83 & 0,63 & 0,52 \\
\hline$U_{\mathrm{m}} \approx 1.4 \mathrm{~m} / \mathrm{s}$ & 0,80 & 0,60 & 0,50 \\
\hline$U_{\mathrm{m}} \approx 1.6 \mathrm{~m} / \mathrm{s}$ & 0,77 & 0,58 & 0,48 \\
\hline$U_{\mathrm{m}} \approx 1.8 \mathrm{~m} / \mathrm{s}$ & 0,75 & 0,56 & 0,47 \\
\hline$U_{\mathrm{m}} \approx 2 \mathrm{~m} / \mathrm{s}$ & 0,74 & 0,55 & 0,45 \\
\hline
\end{tabular}

Table 6: The dependencies of the $\Delta R$ parameter on the initial droplet temperature $T_{\mathrm{w}}$ of the atomized water flow passing the distance of $1 \mathrm{~m}$ in gasoline combustion products.

\begin{tabular}{|c|c|c|c|}
\hline & $R_{\mathrm{m}} \approx 0.15 \mathrm{~mm}$ & $R_{\mathrm{m}} \approx 0.25 \mathrm{~mm}$ & $R_{\mathrm{m}} \approx 0.35 \mathrm{~mm}$ \\
\hline$T_{\mathrm{w}} \approx 293 \mathrm{~K}$ & 0,71 & 0,52 & 0,45 \\
\hline$T_{\mathrm{w}} \approx 295 \mathrm{~K}$ & 0,8 & 0,59 & 0,48 \\
\hline$T_{\mathrm{w}} \approx 300 \mathrm{~K}$ & 1 & 0,73 & 0,55 \\
\hline$T_{\mathrm{w}} \approx 305 \mathrm{~K}$ & 1 & 0,9 & 0,62 \\
\hline$T_{\mathrm{w}} \approx 310 \mathrm{~K}$ & 1 & 1 & 0,71 \\
\hline$T_{\mathrm{w}} \approx 315 \mathrm{~K}$ & 1 & 1 & 0,81 \\
\hline$T_{\mathrm{w}} \approx 318 \mathrm{~K}$ & 1 & 1 & 0,88 \\
\hline
\end{tabular}

The obtained experimental data (table 1-6) illustrate more significant impact of $R_{\mathrm{d}}\left(R_{\mathrm{m}}\right)$ on the integral characteristics of droplet evaporation in high-temperature gases compared to $U_{\mathrm{d}}\left(U_{\mathrm{m}}\right)$. This result can be explained by the established features of the resistance force of liquid droplets in the counter high-temperature gas flow. With growing of $R_{\mathrm{d}}\left(R_{\mathrm{m}}\right)$, resistance force increases, despite the simultaneous rise of the gravity force of a droplet. Since liquid droplets move in hightemperature gas, the conditions of intensive evaporation are met on their surface (boiling conditions are often reached). This 
leads to the growth of resistance force (the direction of blowing vapor from the droplet surface coincides with the vector of resistance force). As the droplet size increases, the effect of vaporization on resistance force rises significantly nonlinearly [11-13]. There is an increase in the contribution of a sufficiently large group of factors to the change in the conditions of heat and mass transfer in the vicinity of droplets [12-13]: the configuration of the droplet surface (nonsphericity), its position relative to the direction of the circumfluent flow, convective flows inside droplets, nonstationarity of their displacement. With a raise of $U_{\mathrm{d}}\left(U_{\mathrm{m}}\right)$ and $R_{\mathrm{d}}=$ const, resistance force varies nonlinearly [11-13]. However, this change is sufficiently small at $U_{\mathrm{d}}\left(U_{\mathrm{m}}\right)<U_{\mathrm{g}}[11-13]$. As a result, the impact of velocities $U_{\mathrm{d}}$ on the integral characteristics of liquid evaporation is moderate (compared to $\left.R_{\mathrm{d}}\right)$. When droplet velocities rise significantly compared to the counter flow $\left(U_{\mathrm{d}}\left(U_{\mathrm{m}}\right)>>U_{\mathrm{g}}\right)$, the influence of this factor will be definitely determining (compared to $R_{\mathrm{d}}\left(R_{\mathrm{m}}\right)$ ) under the conditions of heat transfer on the "liquid - gas" boundary. However, in this case, the disintegration or fragmentation of droplets may happen. Therefore, in this paper, we did not consider such ranges of $U_{\mathrm{d}}\left(U_{\mathrm{m}}\right)$.

Conducted experiments suggest a good correlation of the dependences of $\Delta R$ on $R_{\mathrm{d}}, U_{\mathrm{d}}, T_{\mathrm{w}}$ for the combustion products of various combustible liquids (gasoline, kerosene, alcohol and acetone).

\section{Conclusion}

The experimental studies allowed us to obtain a fairly good correlation between the dependencies of the integral parameter $\Delta R$ for water droplets on determining factors for the combustion products of a large group of typical combustible liquids. This good correlation was established for droplets with different initial sizes. The differences between the absolute values of $\Delta R$ illustrate the feasibility of individual choice of droplet parameters, in order to implement their evaporation with the required characteristics in the combustion products of flammable liquids. Our experimental studies demonstrated that it is possible to select close parameters for several typical combustible liquids. We can generalize the dependencies above for a group of considered gas-vapor-droplet applications (for example, polydisperse fire extinguishing, treatment of liquids based on water evaporation by flame, etc.).

\section{Acknowledgements}

This work was supported by the Russian Science Foundation (project No. 14-39-00003).

\section{References}

[1] Y. Ra and R. D. Reitz, “A vaporization model for discrete multi-component fuel sprays," Int. J. Heat and Mass Transf, vol. 35, pp. 101-117, 2009.

[2] V. I. Terekhov, V. V. Terekhov, N. E. Shishkin, and K. Ch. Bi, "Heat and mass transfer in disperse and porous media experimental and numerical investigations of nonstationary evaporation of liquid droplets," J. Eng. Phys. Thermophys, vol. 83, no. 5, pp. 883-890, 2010.

[3] S. S. Sazhin, A. E. Elwardany, P. A. Krutitskii, V. Depredurand, G. Castanet, F. Lemoine, E. M. Sazhina, and M. R. Heikal, "Multi-component droplet heating and evaporation: numerical simulation versus experimental data," Int. $J$. Therm. Sci, vol. 50, no. 7, pp. 1164-1180, 2011.

[4] C. N. Young, D. A. Johnson, and E. J. Weckman, "A model-based validation framework for PIV and PTV," Exp. Fluids, vol. 36, no. 1, pp. 23-35, 2004.

[5] E. Y. Stepanov, V. P. Maslov, and D. L. Zakharov, "A Stereo PIV system for measuring the velocity vector in complex gas flows," Meas. Tech., vol. 52, no. 6, pp. 626-631, 2009.

[6] C. del Pino, L. Parras, M. Felli, and R. Fernandez-Feria, "Structure of trailing vortices: Comparison between particle image velocimetry measurements and theoretical models," Phys. Fluids, vol. 23, pp. 013602, 2011.

[7] T. Hadad and R. Gurka, "Effects of particle size, concentration and surface coating on turbulent flow properties obtained using PIV/PTV," Exp. Therm Fluid Sci., vol. 45, pp. 203-212, 2013.

[8] N. Takagaki and S. Komori, "Air-water mass transfer mechanism due to the impingement of a single liquid drop on the air-water interface," Int. J. Multiph. Flow, vol. 60, pp. 30-39, 2014.

[9] R. S. Volkov, G. V. Kuznetsov, and P. A. Strizhak, "Experimental investigation of mixtures and foreign inclusions in water droplets influence on integral characteristics of their evaporation during motion through high-temperature gas area," Int. J. Therm. Sci., vol. 88., pp. 193-200, 2015.

[10] G. V. Kuznetsov, P. A. Kuibin, and P. A. Strizhak, "Estimation of the numerical values of the evaporation constants of 
the water drops moving in the high temperature gas flow," High Temp., vol. 53, no. 2, pp. 254-258, 2015.

[11] O. V. Vysokomornaya, G. V. Kuznetsov, and P. A. Strizhak, "Heat and mass transfer in the process of movement of water drops in a high-temperature gas medium," J. Eng. Phys. Thermophys., vol. 86, pp. 62-68, 2013.

[12] P. A. Strizhak, "Influence of droplet distribution in a "water slug" on the temperature and concentration of combustion products in its wake," J. Eng. Phys. Thermophys., vol. 86, no. 4, pp. 895-904, 2013.

[13] D. O. Glushkov, G. V. Kuznetsov, and P. A. Strizhak, "Numerical investigation of water droplets shape influence on mathematical modeling results of its evaporation in motion through a high-temperature gas," Math. Problems Eng., vol. 2014, 2014. 